\title{
UTILIZAÇÃO DOS MACROINVERTEBRADOS BENTÔNICOS COMO INDICADORES DA QUALIDADE DA ÁGUA NO CÓRREGO RETIRO SAUDOSO, EM RIBEIRÃO PRETO - SP
}

\author{
Marcela Del Lama Kamada ${ }^{1}$
}

Gisele Maria de Lucca ${ }^{2}$

José Valdecir de Lucca $^{3}$

RESUMO: O objetivo desse trabalho foi avaliar a qualidade da água no Córrego Retiro Saudoso em Ribeirão Preto (SP), utilizando os macroinvertebrados como bioindicadores. Para isso foi utilizado o índice Biological Monitoring Working Party (BMWP) e a relação $\mathrm{O} / \mathrm{O}+\mathrm{C}$. Estudo com macroinvertebrados bentônicos são importantes devido ao papel desses seres no funcionamento dos ecossistemas aquáticos. São considerados bons indicadores e marcadores da integridade biológica, além de participar no fluxo de energia e na ciclagem de nutrientes. Foram selecionados dois pontos distintos em duas campanhas comparativas, sendo uma realizada no mês de julho (período seco) e outra no mês de outubro (período chuvoso), ambas no ano de 2011. Os organismos foram coletados com um amostrador do tipo Surber (análise quantitativa) e Rede em D (análise qualitativa) e os espécimes preservados em álcool 70\%. Em seguida foram identificados com auxílio de estereomicroscópio óptico e bibliografia especializada. № total, foram coletados 1912 indivíduos e identificados 26 táxons. Os grupos mais representativos da comunidade bentônica foram: Oligochaeta e Chironomidae que juntos representaram 60,0\% da fauna total. Os valores obtidos relação O/O+C e os "scores" do índice BMWP sugerem que os 2 pontos encontram-se impactados. Os resultados da pesquisa auxiliarão em futuros estudos, pois trata-se de uma ferramenta para 0

\footnotetext{
${ }^{1}$ Graduando em Ciências Biológicas pelo Centro Universitário Barão de Mauá. marceladellama@hotmail.com

${ }^{2}$ Graduando em Ciências Biológicas pelo Centro Universitário Barão de Mauá. gi_lucca@hotmail.com

${ }^{3}$ Dr. em Ciências da Engenharia Ambiental pela USP. E-mail.jvlucca@bol.com.br
} 
monitoramento do córrego, além de contribuir com o conhecimento da biodiversidade no estado de São Paulo.

Palavras-chave: Macroinvertebrados bentônicos, índice BMW, Córrego Retiro Saudoso.

\section{INTRODUÇÃO}

O monitoramento de ambientes aquáticos por meio da utilização de organismos vivos é conhecido como biomonitoramento e serve para avaliar mudanças ocorridas no ambiente, geralmente causadas por ação antrópica (BUSS et al., 2003 apud AMORIM e CASTILHO, 2009). Neste sentido os macroinvertebrados bentônicos têm adquirido caráter essencial nos trabalhos de avaliação de impactos sobre os ecossistemas aquáticos (SILVEIRA e QUEIROZ, 2006 apud BIASI et al., 2010). Estes organismos além de estarem presentes em todos os tipos de ecossistemas aquáticos são geralmente mais permanentes, pois vivem de semanas a alguns meses no sedimento. $O$ uso dos macroinvertebrados como bioindicadores é baseado em um princípio simples: submetidos às condições adversas eles morrem ou se adaptam. Neste caso, os organismos que vivem em um dado ecossistema estão adaptados as suas condições ambientais e, por isso, devem refletir o nível de preservação ambiental ou suas alterações provocadas pela emissão de poluentes ambientais (CALLISTO et al., 2005). Além disso, os macroinvertebrados bentônicos têm recebido maior atenção devido à sua importância nos processos ecológicos, por meio da participação no fluxo de energia e ciclagem de nutrientes nos sistemas aquáticos, desempenhando papel importante na troca de fósforo e nitrogênio entre o sedimento e a água de interface (GARDNER et al. 1983 apud LUCCA, 2002; FUKUHARA e SAKAMOTO, 1988 apud LUCCA, 2002). No presente estudo foi aplicado o índice BMWP e a relação $\mathrm{O} / \mathrm{O}+\mathrm{C}$ na avaliação da qualidade da água em 2 pontos do córrego Retiro Saudoso em Ribeirão Preto, SP.

Criada em 1976 a ferramenta BMWP é um dos índices mais utilizados em todo o mundo, este índice ordena as famílias de macroinvertebrados aquáticos em 9 grupos, seguindo um gradiente de menor a maior tolerância dos organismos à poluição orgânica. O índice BMWP baseia-se no somatório de valores de tolerância (sensibilidade a 
poluentes orgânicos) atribuídos a cada grupo de macroinvertebrado de acordo com sua capacidade de sobreviver em diferentes situações de qualidade de água. Este é um índice qualitativo e leva em conta a presença ou ausência de famílias (BISPO et al, 2006; FERNANDES, 2007). O índice BMWP foi sendo adaptado de acordo com a região/local de estudo. Devido a essas características o biomonitoramento, com emprego da fauna macrobentônica como bioindicadora de qualidade de água, apresenta uma clara vantagem sobre a avaliação físico-química tradicional, não só em função do baixo custo dos equipamentos e técnicas empregadas como também por fornecer resultados rápidos. Já a relação Oligochaeta/Oligochaeta + Chironomidae é representada por valores que variam de 0 a 1 sendo que quanto mais próximos a 1 a relação, mais impactado é o local. Essa relação pode ser aumentada com o enriquecimento orgânico (HERGENRADER E LESSIG, 1980), levando a eutrofização da água.

\section{MATERIAL E MÉTODOS}

\section{1 Área de estudo}

A bacia do Córrego Retiro Saudoso situa-se no município de Ribeirão Preto - SP e possui área de aproximadamente $13 \mathrm{~km}$ (Figura 1). A sua nascente encontra-se nas proximidades da Rodovia Anhanguera, continua o seu percurso pela Rua Maurílio Biagi, em seguida, na Avenida Dr. Francisco Junqueira. O Córrego Retiro Saudoso encontra-se com o seu afluente, Ribeirão Preto, no cruzamento entre a Rua Jerônimo Gonçalves e Av. Dr. Francisco Junqueira. A cidade de Ribeirão Preto está localizada no Nordeste do Estado de São Paulo. A área total do município é de 650,00 km² e abrange 614.759 habitantes. O clima da cidade é tropical com verão chuvoso e inverno seco. A temperatura média é de $25^{\circ} \mathrm{C}$ no verão e $19^{\circ} \mathrm{C}$ no inverno. A precipitação pluviométrica média anual é de 1.426,80 mm de chuva. Segundo dados do Departamento de Água e Esgoto de Ribeirão Preto (DAERP), o Córrego é todo canalizado. 


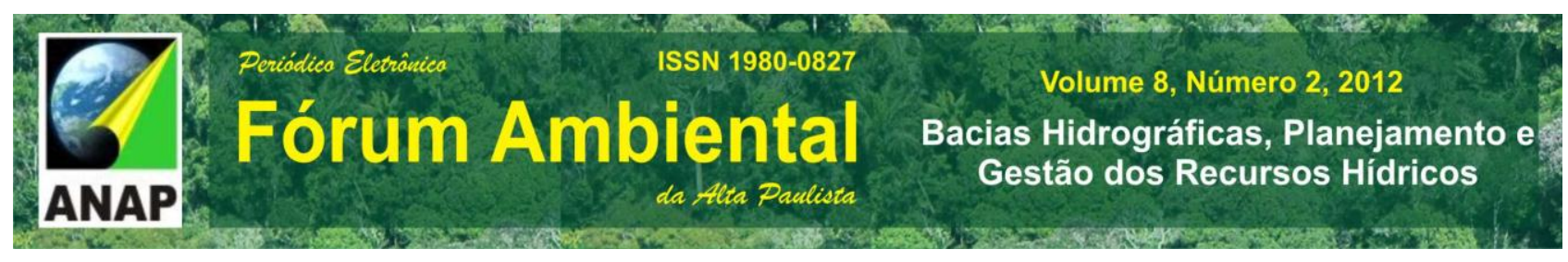

Ao longo do ano de 2011, foram realizadas duas coletas: uma no período de chuva (outubro) e outra no período de seca (julho), ambas no Córrego Retiro Saudoso, porém, em pontos distintos.

O ponto 1, encontra-se próximo à nascente, segundo as seguintes coordenadas

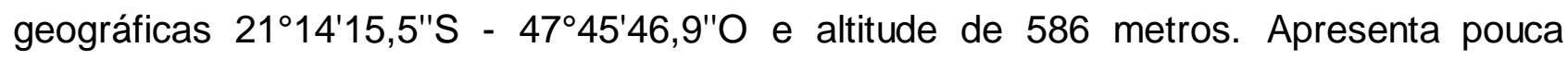
vegetação e início de um reflorestamento ao seu entorno, provavelmente para compensação ambiental da construção de um condomínio a sua borda. $\mathrm{O}$ ponto 2 , encontra-se nas proximidades da área urbanizada do centro da cidade de Ribeirão Preto,

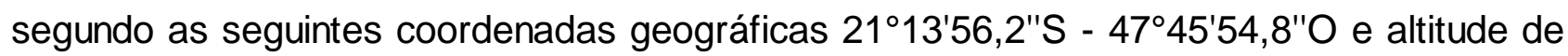
567 metros. Apresenta reflorestamento ao longo do seu percurso. Para se obter maior precisão, os pontos foram georeferenciados com GPS da marca Garmim modelo Etrex.

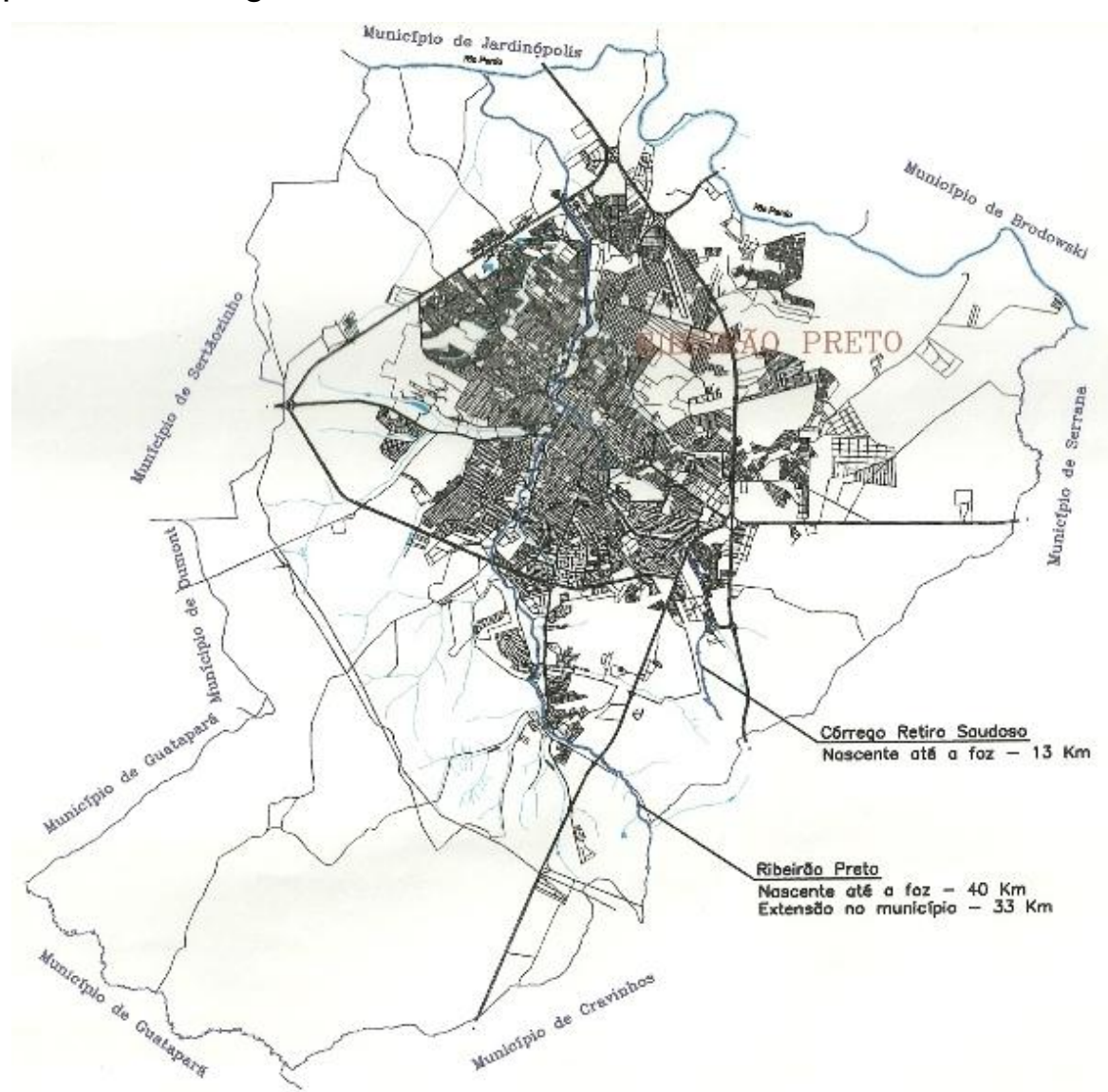

Figura 1: Caracterização dos pontos de amostragem

Fonte: Departamento de Água e Energia Elétrica (DAEE), 2011

\subsection{Coleta e análise dos macroinvertebrados bentônicos}


Foi utilizado um amostrador surber com $0,09 \mathrm{~m}^{2}$ de área amostral em tréplica para análise quantitativa e utilizou-se a rede em $D$ fazendo a varredura para idem qualitativa, as amostras foram fixadas com formol $8 \%$ e armazenadas em saco plástico, no local da coleta. No laboratório utilizou-se uma solução de rosa bengala, com a finalidade de corar os organismos presentes na amostra e facilitar sua visualização e separação do restante do material. Essa solução foi deixada no material durante aproximadamente um dia para efeito de coloração. Para triagem dos organismos bentônicos foi utilizada uma bandeja transluminada. Após a triagem, os organismos foram armazenados em frascos e preservados no álcool a $70 \%$.

\subsection{Tratamento dos dados}

Foi utilizada a metodologia BMWP proposta por Junqueira e Campos (1998) para avaliação da qualidade da água. Esse índice atribui valores (scores) para cada família com base na sua tolerância ao impacto. Os valores variam entre 1 e 10 e são atribuídos de acordo com a sensibilidade das famílias a poluentes orgânicos. Famílias sensíveis a altos níveis de poluentes recebem valores mais altos, enquanto famílias tolerantes recebem valores mais baixos.

Tabela 1: Valores de referência dos Índices BMWP CETEC

\begin{tabular}{|c|c|c|c|}
\hline Classe & Qualidade & BMWP & Significado \\
\hline 1 & Excelente & $>81$ & águas muito limpa \\
\hline 2 & Boa & $61-80$ & $\begin{array}{l}\text { águas pouco } \\
\text { contaminada }\end{array}$ \\
\hline 3 & Regular & $41-60$ & $\begin{array}{c}\text { águas } \\
\text { contaminadas }\end{array}$ \\
\hline 4 & Ruim & $26-40$ & $\begin{array}{l}\text { águas muito } \\
\text { contaminada }\end{array}$ \\
\hline 5 & Péssima & $<25$ & $\begin{array}{c}\text { águas fortemente } \\
\text { contaminadas }\end{array}$ \\
\hline
\end{tabular}


A relação Oligochaeta/Chironomidae proposta por Wiederholm (1980), pode ser calculada a partir da seguinte fórmula:

$$
(\mathrm{O} / \mathrm{O}+\mathrm{C})
$$

Onde: $\mathbf{O}$ corresponde ao número total de Oligochaeta e $\mathbf{C}$, ao número total de Chironomidae.

Os resultados variam de 0 a 1 , portanto valores maiores determinam maior grau de eutrofização do ambiente.

\section{RESULTADOS E DISCUSSÃO}

O crescimento demográfico e o desenvolvimento dos centros urbanos, industriais e agrícolas próximos aos ambientes aquáticos têm sido apontados como principais fatores da acelerada deterioração da qualidade da água (SPERLING, 1993; MORAES e JORDÃO, 2002).

As tabelas 2 e 3 apresentam a composição taxonômica (presença e ausência), a riqueza de táxons dos organismos coletados, nos diferentes pontos a abundância absoluta e relativa, respectivamene. De maneira geral, os representantes da Classe Oligochaeta e os da família de Chironomidae foram os mais abundantes nos dois períodos. Chironomidae é um dos grupos mais populosos, sendo predominante em vários sistemas estudados (MARQUES et al., 1999). Sendo assim, altas densidades dessa família podem evidenciar elevado teor de matéria orgânica no ambiente (MARQUES et al., 1999), o que os tornam eficientes indicadores de degradação ambiental. Com relação aos Oligochaeta é uma das classes de macroinvertebrados bentônicos mais importantes pois sua presença tem sido reportada por estarem presentes em ambientes organicamente poluídos (MILBRINK, 1994; LANG, 1997; 1998) sendo considerados bons indicadores ambientais (WETZEL, 1983). Neste sentido, o registro de elevada densidade desses anelídeos indica um sistema impactado e eutrofizado. 
Tabela 2: Classificação taxonômica e riqueza de táxons nos pontos P1 e P2 em julho e outubro de 2011.

\begin{tabular}{lllll}
\hline & JULHO & & \multicolumn{2}{l}{ OUTUBRO } \\
Táxon & P1 & P2 & P1 & P2 \\
\hline Melanoides tuberculata & + & + & - & + \\
Planorbidae & - & + & - & + \\
Physidae & - & + & - & - \\
Thiaridae & - & + & - & + \\
Corbiculidae & + & + & + & + \\
Baetidae & + & + & + & + \\
Elmidae & + & + & + & - \\
Dytiscidae & - & - & + & - \\
Scarabaeidae & - & - & - & + \\
Gomphidae & + & + & + & + \\
Calopterigidae & - & & + & - \\
Chironomidae & + & + & + & + \\
Hydrophychidae & - & + & - & + \\
Oligochaeta & + & + & + & + \\
Nematoda & - & + & + & + \\
Simuliidae & + & + & + & + \\
Hydrobiidae & - & + & - & + \\
Vellidae & - & - & + & + \\
Libelullidae & - & - & - & - \\
Leptophebiidae & - & - & + & - \\
Glossossomatidae & + & - & - & - \\
Leptoceridae & - & - & - & + \\
Hydroptilidae & - & - & - & + \\
Hirudinea & - & - & - & + \\
Platyhelminthes & - & - & - & + \\
Pyralidae & - & - & - & + \\
\hline Riqueza de táxons & 10 & 14 & 12 & + \\
\hline & & & & + \\
\hline & + & - & + \\
\hline
\end{tabular}




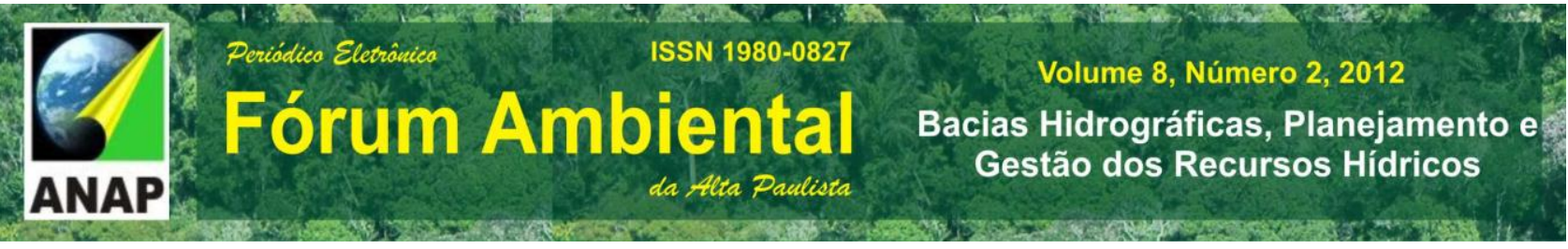

Tabela 3: Classificação taxonômica, abundância absoluta (AA) relativa (AR\%) dos macroinvertebrados bentonicos nos pontos P1 e P2 em julho e outubro de 2011.

\begin{tabular}{|c|c|c|c|c|c|c|c|c|}
\hline \multirow{2}{*}{ Táxon } & \multicolumn{4}{|c|}{ Julho 2011} & \multicolumn{3}{c|}{ Outubro de 2011 } \\
\cline { 2 - 9 } & \multicolumn{2}{|c|}{ Ponto 1 } & \multicolumn{2}{c|}{ Ponto 2 } & \multicolumn{2}{c|}{ Ponto 1 } & \multicolumn{2}{c|}{ Ponto 2 } \\
\cline { 2 - 9 } & AA & AR \% & AA & AR \% & AA & AR \% & AA & AR \% \\
\hline Melanoides tuberculata & 2 & 0,79 & 9 & 2,35 & 0 & 0,00 & 12 & 1,44 \\
\hline Planorbidae & 0 & 0,00 & 24 & 6,27 & 0 & 0,00 & 118 & 14,15 \\
\hline Physidae & 0 & 0,00 & 29 & 7,57 & 0 & 0,00 & 0 & 0,00 \\
\hline Thiaridae & 0 & 0,00 & 8 & 2,09 & 0 & 0,00 & 9 & 1,08 \\
\hline Corbicula flumínea & 1 & 0,40 & 38 & 9,92 & 140 & 31,89 & 113 & 13,55 \\
\hline Baetidae & 8 & 3,17 & 1 & 0,26 & 49 & 11,16 & 26 & 3,12 \\
\hline Elmidae & 1 & 0,40 & 1 & 0,26 & 4 & 0,91 & 0 & 0,00 \\
\hline Dytiscidae & 0 & 0,00 & 0 & 0,00 & 1 & 0,23 & 0 & 0,00 \\
\hline Scarabaeidae & 0 & 0,00 & 0 & 0,00 & 0 & 0,00 & 1 & 0,12 \\
\hline Gomphidae & 4 & 1,59 & 5 & 1,31 & 3 & 0,68 & 1 & 0,12 \\
\hline Calopterigidae & 0 & 0,00 & 3 & 0,78 & 1 & 0,23 & 0 & 0,00 \\
\hline Chironomidae & 10 & 3,97 & 21 & 5,48 & 38 & 8,66 & 89 & 10,67 \\
\hline Hydrophychidae & 0 & 0,00 & 10 & 2,61 & 0 & 0,00 & 24 & 2,88 \\
\hline Oligochaeta & 222 & 88,10 & 217 & 56,66 & 194 & 44,19 & 329 & 39,45 \\
\hline Nematoda & 0 & 0,00 & 1 & 0,26 & 3 & 0,68 & 15 & 1,80 \\
\hline Simuliidae & 2 & 0,79 & 3 & 0,78 & 4 & 0,91 & 20 & 2,40 \\
\hline Hydrobiidae & 0 & 0,00 & 13 & 3,39 & 0 & 0,00 & 55 & 6,59 \\
\hline Vellidae & 0 & 0,00 & 0 & 0,00 & 1 & 0,23 & 2 & 0,24 \\
\hline Libelullidae & 1 & 0,40 & 0 & 0,00 & 0 & 0,00 & 0 & 0,00 \\
\hline Leptophebiidae & 0 & 0,00 & 0 & 0,00 & 1 & 0,23 & 0 & 0,00 \\
\hline Glossossomatidae & 1 & 0,40 & 0 & 0,00 & 0 & 0,00 & 0 & 0,00 \\
\hline Leptoceridae & 0 & 0,00 & 0 & 0,00 & 0 & 0,00 & 8 & 0,96 \\
\hline Hydroptilidae & 0 & 0,00 & 0 & 0,00 & 0 & 0,00 & 1 & 0,12 \\
\hline Hirudinea & 0 & 0,00 & 0 & 0,00 & 0 & 0,00 & 9 & 1,08 \\
\hline Platyhelminthes & 0 & 0,00 & 0 & 0,00 & 0 & 0,00 & 1 & 0,12 \\
\hline Pyralidae & 0 & 0,00 & 0 & 0,00 & 0 & 0,00 & 1 & 0,12 \\
\hline Riqueza de táxons & 252 & & 383 & & 439 & & 834 & \\
\hline & & & & & & & & 0 \\
\hline
\end{tabular}

A relação Oligochaeta/Chironomidae foi calculada, a fim de classificar o grau trófico da água. Na tabela 4, são apresentados os resultados obtidos nos diferentes pontos, durante o mês de Julho e Outubro de 2011. O alto valor obtido na relação Oligochaeta/Chironomidae (valores próximos a 1) demonstra um ambiente eutrofizado, com alto grau de poluição. Segundo Esteves (1998) a eutrofização é o aumento da 
concentração de nutrientes, especialmente fósforo e nitrogênio, nos ecossistemas aquáticos, tendo como consequência os aumentos de sua produtividade. A eutrofização artificial é uma reação em cadeia com casos e efeitos bem evidentes. Com o rompimento do estado de equilíbrio, o ecossistema passa a produzir mais matéria orgânica do que é capaz e consumir e decompor, causando um desequilíbrio ecológico que acarreta profundas mudanças no metabolismo de todo o ecossistema. Observa-se durante o processo de eutrofização artificial, no que se refere à concentração de nutrientes, um aumento da concentração de quase todos os elementos químicos essenciais à produtividade primária. O aumento na concentração de cada nutriente geralmente é diferenciado, pois depende da influência a qual o ecossistema está submetido (esgotos domésticos, industriais, atividade agrícola, etc.), sendo o fosfato mais importante nesse processo de eutrofização artificial. Provavelmente está sendo lançado no córrego esgoto "in natura" próximo aos pontos de coleta.

Essa relação pode ser aumentada com 0 enriquecimento orgânico (HERGENRADER \& LESSIG, 1980), levando a eutrofização da água.

Tabela 4: Valores da relação O/O+C nos pontos de amostragem em julho e outubro de 2011.

\begin{tabular}{ccc}
\hline Mês coleta & Ponto & $(\mathbf{O} / \mathbf{O}+\mathbf{C})$ \\
\hline Julho/2011 & P1 & 0,95 \\
& P2 & 0,91 \\
Outubro/2011 & P1 & 0,84 \\
& P2 & 0,79 \\
\hline
\end{tabular}

Na tabela 5 é apresentada a classificação da qualidade da água nos pontos 1 e 2 em julho e outubro de 2011 utilizando o índice BMWP - CETEC através dos "scores" das famílias dos macroinvertebrados bentônicos registrados no presente trabalho.

A água do Córrego Retiro Saudoso, segundo o índice BMWP-CETEC, é classificada como ruim e regular em todos os pontos durante o período estudado.

O monitoramento biológico é realizado principalmente por meio da aplicação de diferentes protocolos de avaliação, índices biológicos e multimétricos, tendo como base a utilização de bioindicadores de qualidade de água e habitat. As famílias Oligochaeta e Chironomidae respectivamente foram os mais abundantes, predominando em todos os 
pontos de amostragem. Comparando os resultados obtidos no índice BMWP com a relação Oligochaeta/Chironomidae, podemos deduzir que o Córrego apresenta águas de qualidade regular a ruim, devido à grande presença de organismos resistentes e o menor aparecimento de espécies sensíveis. Desse modo os dois pontos encontram-se impactados.

Neste sentido recomenda-se o monitoramento com os macroinvertebrados bentônicos no Córrego Retiro Saudoso em Ribeirão Preto, SP.

Tabela 5: BMWP "score", classificação e significado nos pontos 1 e 2 em julho e outubro de 2011.

\begin{tabular}{|c|c|c|c|}
\hline Local/Data & BMWP "Score" & Classificação & Significado \\
\hline P1 jul.11 & 38 & Ruim & Agua muito contaminada \\
\hline P2 jul.11 & 43 & regular & Agua contaminada \\
\hline P1 out.11 & 42 & regular & Agua contaminada \\
\hline P2 out.11 & 50 & regular & Agua contaminada \\
\hline
\end{tabular}

\section{CONCLUSÃO}

- Conclui-se que a presença e grande abundância das famílias: Chironomidae e Oligochaeta influenciam nos resultados do índice BMWP e na relação Oligochaeta/Chironomidae, devido à resistência e tolerância desses organismos a diferentes habitats.

- A utilização dos macroinvertebrados bentônicos como bioindicadores por meio da aplicação do índice BMWP e da relação $\mathrm{O} / \mathrm{O}+\mathrm{C}$ foi eficiente na avaliação da qualidade da água do Córrego Retiro Saudosos em Ribeirão Preto SP, caracterizando-o como eutrofizado e poluído.

\section{REFERÊNCIAS}

AAGAARD, K. The chironomid fauna of North Norwegian lakes with a discussion on the methods of community classification. Hol. Ecol., Copenhagen, v.9, p.1-12, 1986. 
AMORIM, A. C. F.; CASTILHO, A. R. Macroinvertebrados bentônicos como Bioindicadores da qualidade da água do Baixo Rio Perequê, Cubatão, São Paulo, Brasil. Biodiversidade Pampeana, v.7, n.12, p.16-22, 2009. Disponível em<http://www.revistaseletronicas. pucrs.br/ojs/index.php/biodiversidadepampeana>. Acesso em: 30 mar 2011.

Barnes, R.S.K., Calow, P. \& Olive, P.J.W. 1995. Os invertebrados: uma nova síntese. São Paulo: Atheneu Editora. p. 118-121.

BIASI, C. et al. Biomonitoramento das águas pelo uso de macroinvertebrados bentônicos: oito anos de estudo em riachos da região do Alto Uruguai (RS). Perspectiva, Erechim, v.34, n.125, p. 67-77, 2010. <http://www.uricer.edu.br/new/site/pdfs/perspectiva/125_75.pdf >. Acesso em: 27 mar 2011

BISPO, P.C.; OLIVEIRA, L.G. Diversity and structure of Ephemeroptera, Plecoptera and Trichoptera (Insecta) assemblages from riffles in mountain streams of Central Brazil. Rev. Bras Zool., Curitiba, v.24, n. 2, jun. 2006.

CALLISTO, M.; GOULART, M. Invertebrate drift along a longitudinal gradient in a Neotropical stream in Serra do Cipó National Park, Brazil. Hydrobiologia, 539: p. 47-56. 2005.

CALLISTO, M.; MORETTI, M.; GOULART, M. Macroinvertebrados bentônicos como ferramenta para avaliar a saúde de riachos. Revista Brasileira de Recursos Hídricos: v. 6, p. 71-82, jan/mar 2001.

ESTEVES, F. A. Fundamentos de limnologia. 2 ed. Rio de Janeiro: Interciência, 1998.

Fernandes, S. E.; Takeda, A. M.; Fujita, D. S.; Pinha, G. D.; Behrend, R. D. L.; Ragonha, F. H. 2007. Substrato artificial em diferentes canais secundários da planície aluvial do alto rio Paraná: Limnoperna fortunei versus invertebrados aquáticos. Anais do II Encontro Sulamericano de Integração de Ações para o Controle do Mexilhão Dourado, Rio de Janeiro, Brasil, p.16.

Hergenrader, G.L. \& Lessig, D.C. 1980. Eutrophication of the Salt Valley reservaoirs, 1968-73 III. The macroinvertebrate community: its development, composition, and change in response to eutrophication. Hydrobiol. 75:7-25.

JUNQUEIRA, V.M. \& CAMPOS, S.C.M. Adaptation of the "BMWP" method for water quality evaluation to Rio das Velhas watershed (Minas Gerais, Brazil). Acta Limnol. Bras., v.10, p.125-135, 1998.

LANG, C. Constrasting responses of oligochaeta (Annelida) and chironomids (Diptera) to the abatement of eutrophication in Lake Neuchâtel. Aquat. sci, Basel, v. 61, p. 206-214, 1998. 
LUCCA, J. V. Composição, distribuição e abundância dos macroinvertebrados bentônicos da Lagoa do Caçó (MA). 2002. p. 123. Dissertação (Mestrado em ecologia) - Escola de Engenharia de São Carlos - Universidade de São Paulo, São Carlos.

Marques, M. G. S. M.; Ferreira, R. L. \& Barbosa, F. A. R. A comunidade de macroinvertebrados aquáticos e características limnológicas das laqoas carioca e da barra, Parque Estadual do Rio Doce, MG. Revista Brasileira de Biologia 59(2): p. 203210.1999.

MILBRINK, G. Oligochaetes and pollution in two deep Norwegian lakes. Hydrobiologia 278: p. 213-222. 1994

MORAES, D. S. D. L.; JORDÃO, B. Q. Degradação de recursos hídricos e seus efeitos sobre a saúde humana. Rev. Saúde Pública, São Paulo, v.36, n.3, p.370-374. 2002.

SPERLING, E. V. Considerações sobre a saúde de ambientes aquáticos. Revista Bio, Rio de Janeiro, v. 3, p.53-56. 1993.

WETZE, R. G. Limnology. 2 ed. Saunders College Publ, Washington, p. 919. 1983.

WIEDERHOLM, T. Use of benthos in the lake monitoring. J. Wat. Poll. Control. Feder., New York, v.5, n.3, p.537-547, 1980. 